\title{
Acute treatment effects on GFR in randomized clinical trials of kidney disease progression
}

Brendon L Neuen, MBBS MSc${ }^{1}$; Hocine Tighiouart, $\mathrm{MS}^{2}{ }^{3}$; Hiddo JL Heerspink, PharmD ${ }^{4}$; Edward F Vonesh, $\mathrm{PhD}^{5}$; Juhi Chaudhari, $\mathrm{MPH}^{6}$; Shiyuan Miao, $\mathrm{MS}^{6}$; Tak Mao Chan, MD DSc ${ }^{7}$; Fernando C Fervenza, $\mathrm{MD} \mathrm{PhD}^{8}$; Jürgen Floege, $\mathrm{MD}^{9}$; Marian Goicoechea, $\mathrm{MD}, \mathrm{PhD}^{10}$; William G Herrington, $\mathrm{MD}^{11}$; Enyu Imai, MD ${ }^{12}$; Tazeen H Jafar, MD MPH ${ }^{13}{ }^{14}$; Julia B Lewis, MD ${ }^{15}$; Philip Kam-Tao Li, MD ${ }^{16}$; Francesco Locatelli, MD ${ }^{17}$; Bart D Maes, MD PhD ${ }^{18}$; Ronald D Perrone, $\mathrm{MD}^{6}$; Manuel Praga, $\mathrm{MD}^{19}$; Annalisa Perna, MD ${ }^{20}$; Francesco P Schena, MD ${ }^{21}$; Christoph Wanner, MD ${ }^{22}$; Jack FM Wetzels, MD ${ }^{23}$; Mark Woodward, $\mathrm{PhD}^{124}$; Di Xie, $\mathrm{MD}^{25}$; Tom Greene, $\mathrm{PhD}^{26}$; Lesley A Inker, $\mathrm{MD} \mathrm{MS}^{6}$

On behalf of CKD-EPI Clinical Trials

Corresponding author:

Lesley A Inker

Division of Nephrology

Tufts Medical Center

800 Washington St.

Box 391, Boston MA USA 02474

LInker@Tuftsmedicalcenter.org

Word count: 3491 [2550 (excluding methods); abstract (247); significance statement (110)]

Tables and figures: $5(1+4)$

\footnotetext{
${ }^{1}$ The George Institute for Global Health, University of New South Wales, Sydney, Australia

${ }^{2}$ Institute for Clinical Research and Health Policy Studies, Tufts Medical Center, Boston, MA, USA

${ }^{3}$ Tufts Clinical and Translational Science Institute, Tufts University, Boston, MA, USA

${ }^{4}$ Department of Clinical Pharmacy and Pharmacology, University of Groningen, University Medical Center Groningen, Groningen, Netherlands

${ }^{5}$ Department of Preventive Medicine, Division of Biostatistics, Northwestern University, Chicago, IL, USA

${ }^{6}$ Division of Nephrology, Tufts Medical Center, Boston, MA, USA

7 Department of Medicine, University of Hong Kong, Queen Mary Hospital, Pokfulam, Hong Kong

${ }^{8}$ Division of Nephrology and Hypertension and Department of Medicine, Mayo Clinic Rochester, MN, USA

${ }^{9}$ Division of Nephrology, RWTH Aachen University, Aachen, Germany

${ }^{10}$ Department of Nephrology, Hospital General Universitario Gregorio Marañón, Madrid, Spain

${ }^{11}$ Medical Research Council Population Health Research Unit at the University of Oxford,, Nuffield Department of Population Health, University of Oxford, Oxford, UK

12 Nakayamadera Imai Clinic, Takarazuka, Japan

${ }^{13}$ Program in Health Services and Systems Research, Duke-NUS Medical School, Singapore

${ }^{14}$ Duke Global Health Institute, Duke University, Durham, North Carolina

${ }^{15}$ Division of Nephrology, Vanderbilt University, Nashville, TN, USA

${ }^{16}$ Division of Nephrology, Prince of Wales Hospital, Chinese University of Hong Kong, Shatin, Hong Kong

${ }^{17}$ Department of Nephrology, Alessandro Manzoni Hospital ( past Director), ASST Lecco, Italy

${ }^{18}$ Department of Nephrology, AZ Delta, Roeselare, Belgium

${ }^{19}$ Nephrology Department, Hospital Universitario 12 de Octubre, Madrid, Spain

${ }^{20}$ Istituto di Ricerche Farmacologiche Mario Negri IRCCS, Bergamo, Italy

${ }^{21}$ Renal, Dialysis and Transplant Unit, Department of Emergency and Organ Transplantation, University of Bari, Bari, Italy

22 Division of Nephrology, University Hospital of Würzburg, Würzburg, Germany

${ }^{23}$ Department of Nephrology, Radboud University Medical Center, Radboud Institute for Health Sciences, Nijmegen, The Netherlands

${ }^{24}$ The George Institute for Global Health, School of Public Health, Imperial College London, UK

25 Division of Nephrology, Nanfang Hospital, Southern Medical University, Guangzhou, China

${ }^{26}$ Division of Epidemiology, Department of Internal Medicine, University of Utah, Salt Lake City, UT, USA
} 


\section{SIGNIFICANCE STATEMENT}

Glomerular filtration rate (GFR) slope has been proposed as a surrogate endpoint for progression to kidney failure in chronic kidney disease (CKD) trials. Acute - or immediate- effects on GFR following treatment initiation may complicate the interpretation of long-term treatment effects. In this large meta-analysis of 53 randomized clinical studies of CKD progression, we described the magnitude and nature of the acute effects. Negative acute effects (i.e. an acute reduction in GFR) were observed in renin-angiotensin system blockade and blood pressure lowering trials, while positive acute effects were more common in trials of immunosuppressive therapies. This information can inform the optimal design and analysis plan for randomized clinical trials in CKD. 


\section{ABSTRACT}

Background Acute changes in glomerular filtration rate (GFR) can occur following initiation of interventions for chronic kidney disease (CKD) progression. These complicate the interpretation of treatment effects on long term progression of (CKD). We sought to assess the magnitude and consistency of acute effects in randomized clinical trials (RCT) and explore factors that might impact them.

Methods We performed a meta-analysis of 53 RCTs for CKD progression enrolling 56,413 participants that had at least one estimated GFR measurement by six months following randomization. We defined acute treatment effects as the mean difference in GFR slope from baseline to 3 months between randomized groups. We performed univariable and multivariable meta-regression to assess the impact of intervention type, disease state, baseline GFR and albuminuria on the magnitude of acute effects.

Results The mean acute effect across all studies was $-0.21 \mathrm{~mL} / \mathrm{min} / 1.73 \mathrm{~m}^{2}(95 \% \mathrm{Cl}-0.63$ to 0.22$)$ over three months, with substantial heterogeneity across interventions (95\% coverage interval across studies, -2.50 to $+2.08 \mathrm{~mL} / \mathrm{min} / 1.73 \mathrm{~m}^{2}$ ). Negative average acute effects were observed in renin angiotensin system blockade, blood pressure lowering and sodium-glucose cotransporter 2 inhibitor trials while positive acute effects were observed in trials of immunosuppressive agents. Larger negative acute effects were observed in trials with higher mean baseline GFR.

Conclusion The magnitude and consistency of acute GFR effects varies across different interventions, and is larger at higher baseline GFR. Understanding the nature and magnitude of the acute effects can help inform the optimal design of RCTs in CKD evaluating kidney disease progression. 


\section{Introduction}

A key challenge in the design and conduct of randomized controlled trials (RCTs) of chronic kidney disease (CKD) is that kidney failure typically develops over a long period of time, and thus studies seeking to detect effects on this outcome require substantial follow-up time. As a result, there has been substantial effort from investigators, regulatory authorities, and sponsors towards identifying robust alternative endpoints for kidney failure, particularly for early stages of CKD and for early phase trials. ${ }^{1-6}$

A 2018 scientific workshop convened by the National Kidney Foundation, United States Food and Drugs Administration and European Medicines Agency evaluated the evidence for rate of change in glomerular filtration rate ([GFR] i.e. GFR slope) as an alternative endpoint for kidney disease progression in RCTs. ${ }^{7}$ Based on separate meta-analyses of observational cohorts and RCTs, the workshop concluded that treatment effects on GFR slope accurately predicted treatment effects on clinical outcomes, indicating that GFR slope may be a viable alternative endpoint for kidney disease progression. Based on these data, some ongoing studies are using GFR slope as an endpoint. ${ }^{8-14}$ Interventions that impact CKD progression often produce early, short term effects on GFR (referred to hereon in as acute effects) that differ from their long-term treatment effects (referred to hereon in as chronic slope), as for example are seen with agents that block the renin-angiotensin system (RAS) and sodium glucose cotransporter 2 (SGLT2) inhibitors. ${ }^{15-18}$ The presence of acute effects may complicate the design and interpretation of RCTs in which GFR slope is the primary outcome. For example, negative acute effects may increase risk of falsely concluding no benefit, while positive acute effects may increase the risk of falsely concluding treatment benefit.

While such acute effects are common, there is little understanding of them. We sought to describe the nature and magnitude of acute treatment effects on GFR in RCTs in which kidney disease 
progression was assessed and evaluate the consistency of these effects across key study level characteristics, including intervention type, GFR, and albuminuria.

\section{Methods}

Datasets

As part of our previous work, we developed a pooled database of RCTs by performing a systematic literature search to identify relevant trials and obtaining individual participant data for these studies. ${ }^{1,19-61}$ A complete list of search terms used is provided in Table $\mathbf{S 1}$ and the study inclusion criteria are listed in Table S2. Risks of bias for each study were assessed using the risk-of-bias tool of the Cochrane collaboration ${ }^{62}$ (Figure S1). As we have done previously, we included a separate randomized treatment comparison for each independent treatment versus control comparison for trials that evaluated more than one intervention, but unlike in our prior work we did not pool small trials that had less than 100 participants if the disease and intervention were the same. ${ }^{1,34,42-44,48,49 \text {, }}$ 51-54, 63-65 For the current analysis, we excluded four RCTs that did not have at least one follow-up study visit at or before six months post randomization. ${ }^{63-66}$ The study was approved by Tufts Medical Center Institutional Review Board.

GFR

GFR was estimated using the Chronic Kidney Disease Epidemiology Collaboration (CKD-EPI) 2009 creatinine equation. ${ }^{67}$ Creatinine was standardized to isotope dilution mass spectroscopy traceable reference methods using direct comparison or was reduced by $5 \%$ as has previously been described ${ }^{68}$ 
Our primary definition of the acute treatment effect was the mean difference in the change in GFR from baseline to three months between the treatment and control arms. The primary analysis used analysis of covariance (ANCOVA) to estimate the acute effect while adjusting for baseline GFR as a covariate. This analysis was restricted to studies with baseline GFR and at least one planned measurement of GFR between month 3 and month 6 . For patients who had measurements at 4, 5 , or 6 months, the acute effect was computed at that time. As we have previously performed, we categorized the observed mean acute effect $\left(\mathrm{mL} / \mathrm{min} / 1.73 \mathrm{~m}^{2}\right)$ as very large negative $(-2.5$ or lesser), moderate-to-large negative $(>-2.5$ to $<-1.25)$, small negative $(-1.25$ to $<0)$, small positive $(>0$ to $<1.25)$, moderate-to-large positive $(1.25$ to $<2.5)$ and very large positive ( 2.5 or greater).$^{69}$ In a sensitivity analysis, we fitted a linear mixed effects model to GFR between months 3 and 24 of follow-up, with covariate adjustment for the baseline GFR level. This model includes fixed effects for treatment assignment, baseline GFR and the interactions of these factors with time, as well as random slopes and intercepts for characterizing variation in GFR trajectories across patients. For studies that had follow-up longer than 24 months, the follow-up time was truncated at 24 months to ensure that the long-term trajectory did not overly influence the estimation of the acute effect. This sensitivity analysis was able to formally evaluate mean GFR change to 3 months even if the initial GFR follow-up assessment occurred later than month 3 based on the assumption of linear mean decline between months 3 and 24 . With the exception of the analyses that related the size of the acute effects to baseline GFR and UACR levels (see below), acute effects were estimated based on change in GFR on the linear (raw) scale and expressed in units of $\mathrm{mL} / \mathrm{min} / 1.73 \mathrm{~m}^{2}$.

\section{Meta-Analyses}

We performed separate random effects meta-analyses to model the distribution of "true" treatment effects on the acute GFR change to 3 months across all studies and then separately for subgroups of studies based on intervention type. Our random effects models assumed that the acute effects were normally distributed across studies. We used these models to obtain the mean acute effect across 
the studies included in each analysis with a $95 \%$ confidence interval. In addition, to assess heterogeneity of acute effects between studies we computed $95 \%$ coverage intervals from the mean and the between-study standard deviation of the acute effects from the random-effects metaanalysis. The coverage interval provides lower and upper limits that included $95 \%$ of the acute effects across the studies under the assumption that the acute effects are normally distributed.

We performed separate univariable meta-regression analyses to explore the impact of mean baseline GFR and median baseline urinary albumin to creatinine ratio (UACR) on the magnitude of the acute effects across studies. For these analyses, we analysed the longitudinal GFR measurements for estimation of the acute effect on both on the linear scale and natural log scale. When the longitudinal GFR measurements were expressed on the linear scale, the acute effect is expressed in absolute units of $\mathrm{mL} / \mathrm{min} / 1.73 \mathrm{~m}^{2}$. When the longitudinal GFR measurements were expressed on the natural log scale, the acute effect is expressed as a relative effect and expressed as a ratio of geometric mean GFR levels between the treatment and control groups. Median baseline UACR was log transformed in these analyses irrespective of whether the longitudinal GFR measurements were expressed on the raw scale or natural log scale. Baseline GFR was expressed on the linear scale when the longitudinal GFR measurements were expressed on the linear scale and on the natural log scale when the longitudinal GFR measurements were log transformed. In addition, to consider whether standard of care at the time of the study affected the magnitude of the acute effects, we also performed separate univariable meta-regression analyses by year of publication.

We also performed multivariable meta-regression to further assess the impact of baseline mean GFR and median UACR on acute effects after adjusting for intervention type, and diabetes status. In additional sensitivity analyses, we analysed acute effects by quartiles of baseline GFR within individual studies to compare associations observed at the study and individual participant levels. 
Analyses were performed using SAS version 9.4 (SAS Institute, Cary, NC) and R 3.16.1 (R Project for Statistical Computing, www.r-project.org).

\section{Results}

We included 53 randomized studies enrolling 56,413 participants that had at least one visit by six months following randomization. Aggregate characteristics of included studies stratified by intervention are summarised in Table 1, Table $\mathbf{S 3}$ (stratified by disease) and Table S4. The median baseline UACR was higher in trials with lower mean baseline GFR, except for immunosuppression studies (Figure S2).

Acute effects overall and by intervention

Across all studies, the mean acute effect (difference in GFR between randomized groups) was -0.21 $\mathrm{mL} / \mathrm{min} / 1.73 \mathrm{~m}^{2}$ over the first three months ( $95 \% \mathrm{Cl}-0.63$ to 0.22 ). There was substantial heterogeneity across studies; the $95 \%$ coverage interval for the acute effect across studies ranged from -2.50 to $+2.08 \mathrm{~mL} / \mathrm{min} / 1.73 \mathrm{~m}^{2}$ (Figure 1, Table S5, Figure $\mathbf{S 3}$ and Figure S4). Results were similar in sensitivity analyses using the simple linear mixed models (Table S5 and Figure S5). For specific intervention types, there is evidence that RAS blockade vs. CCB, RAS blockade vs. control, SGLT2 inhibitors vs. placebo, and intensive blood pressure lowering led to negative average acute effects, and that immunosuppressive agents led to a positive average acute effect (Figure 1, Table S5 and Figure S4), although in most cases statistical significance was not reached. The substantial variation in acute effects across different trials persisted within the individual treatment comparison classes (Table S5 and Figure S4), and by year of publication (Figure S6). Heterogeneity was greatest among immunosuppression (95\% coverage interval across studies, -2.34 to $6.29 \mathrm{~mL} / \mathrm{min} / 1.73 \mathrm{~m}^{2}$ ) and RASB vs CCB trials ( $95 \%$ coverage interval across studies, -4.28 to $1.07 \mathrm{~mL} / \mathrm{min} / 1.73 \mathrm{~m}^{2}$ ). 
Table S6 shows the categories of large, moderate, or small observed mean acute effects overall and by intervention. Overall, moderate to large $\left(>-2.5\right.$ to $\left.<=-1.25 \mathrm{~mL} / \mathrm{min} / 1.73 \mathrm{~m}^{2}\right)$ or very large $(<=-2.5$ $\left.\mathrm{mL} / \mathrm{min} / 1.73 \mathrm{~m}^{2}\right)$ negative acute effects were observed in 13 trials, moderate to large $(>=1.25$ to $<2.5$ $\left.\mathrm{mL} / \mathrm{min} / 1.73 \mathrm{~m}^{2}\right)$ or very large $\left(\underline{\Delta=2.5} \mathrm{~mL} / \mathrm{min} / 1.73 \mathrm{~m}^{2}\right)$ positive acute effects were observed in 13 trials, and small positive or negative acute effects were observed in the remaining studies. Reflecting a combination of true variation in acute effects and random sampling error which predominates in smaller trials, in these descriptive analyses negative acute effects were observed in 4 out of 4 studies comparing RAS blockade to CCBs, in 13 out of 23 studies comparing RAS blockade to control, in 1 trial comparing SGLT2 inhibitors to control, and 4 out of 5 trials of immunosuppressive agents. Positive acute effects were observed in 11 of the 15 trials of immunosuppressive agents (Table S6).

\section{Acute effects by baseline GFR}

Larger negative acute effects (expressed in units of $\mathrm{ml} / \mathrm{min} / 1.73 \mathrm{~m}^{2}$ ) tended to be observed in studies with higher levels of mean baseline GFR ( $P=0.019$; Figure 2). The association between the acute effect and baseline GFR remained mostly consistent after adjustment for intervention and diabetic status at the study level (Table S7). For trials of RAS blockade versus control, the mean acute effect (SE) varied from $0.24(0.36)$ when the mean baseline GFR was $20 \mathrm{ml} / \mathrm{min} / 1.73 \mathrm{~m}^{2}$ compared to -1.57 (0.42), when mean baseline GFR was $80 \mathrm{~mL} / \mathrm{min} / 1.73 \mathrm{~m}^{2}$ (Figure 3). For trials comparing low versus usual BP control the mean acute effect varied from $0.24(0.43)$ when mean baseline GFR was $20 \mathrm{~mL} / \mathrm{min} / 1.73 \mathrm{~m}^{2}$ compared to -2.25 (0.55) when mean baseline GFR was 80 $\mathrm{mL} / \mathrm{min} / 1.73 \mathrm{~m}^{2}$ (Figure 3). There was no clear association between the acute effect and mean baseline GFR for RAS blockade vs calcium-channel blockers or for immunosuppression trials (Figure 3), although the wide confidence bands indicate low statistical power for these analyses. The association between the acute effect and baseline GFR did not substantively change after removing trials of immunosuppressive agents (data not shown). The effect persisted when GFR was log transformed (Figure S7 and Figure S8). For RAS blockade vs control, there was a similar relationship 
between the acute effect and baseline GFR when participants were categorized by GFR quartiles within individual studies (Figure S9).

Acute effects by baseline UACR

Acute effects were also somewhat more negative for studies with lower baseline UACR (Figure 2), although there was an attenuation of the effect after adjustment for baseline GFR (Table S7). When stratified by intervention type, the association between the acute effect and baseline UACR was only observed in studies evaluating low versus usual blood pressure targets, with no association observed between baseline UACR and the magnitude of acute effects for other interventions (Figure 4). Results were similar in sensitivity analyses when the acute effect was estimated based on log transformed GFR (Figure S7 and Figure S8).

\section{Discussion}

We provide a comprehensive assessment of the magnitude and consistency of acute treatment effects on GFR across a range of interventions and disease states in 53 RCTs evaluating treatments for CKD progression. Negative acute effects were observed in the majority of RAS blockade, blood pressure lowering and SGLT2 inhibitor trials, while positive acute effects were observed in immunosuppression trials. However, even within interventions there was substantial variability in the observed acute effects. These findings have important implications for the design of clinical trials assessing kidney disease progression and highlight the importance of understanding the nature and magnitude of acute effects for specific interventions in early-phase trials to inform the design and analysis of longer-term studies, specifically, the decision to use a slope-based outcome or a clinical endpoint. 
Negative acute effects are known to be common in interventions for CKD progression. For example, RAS blockade, SGLT2 inhibitors and blood pressure lowering all lead to hemodynamic changes in GFR which are thought to differ from the long-term protective effect on the kidney. These hemodynamic effects influence the function of individual nephrons and not the number of nephrons and are reversible upon treatment discontinuation. Negative acute effects may also be a result of changes in non GFR determinants of creatinine, as in, for example, decreased creatinine secretion by the tubules. Regardless of the mechanism, negative acute effects can increase the risk of false negative conclusions about the treatment benefit. Negative acute effects may also reduce the utility of slopebased analyses or time-to-event analyses with endpoints defined by $30 \%$ or $40 \%$ GFR declines by eliminating power advantages of total GFR slope or time to lesser GFR decline compared with the clinical endpoint. ${ }^{69-71}$ In a post-hoc analysis of the CANVAS Program assessing the effect of the SGLT2 inhibitor canagliflozin on different GFR decline thresholds (i.e. 50, 40 and 30\% declines in GFR), the power advantage of using lesser declines in GFR was only observed after excluding the negative acute hemodynamic effect of canagliflozin. ${ }^{72}$

One potential solution to overcome negative acute effects and utilize slope as an endpoint is to use chronic (rather than total) GFR slope, which computes the change in GFR slope following the acute phase. However, this approach may introduce bias from attenuation of the acute effect over time or early discontinuation of the study treatment. ${ }^{69,73,74}$ Another potential strategy is to assess GFR slope from baseline to off-treatment measures when it is anticipated that acute and reversible hemodynamic effects will no longer be present. Ongoing trials of sparsentan and atresentan in people with focal segmental glomerulosclerosis and IgA nephropathy plan to account for anticipated negative acute effects by employing both approaches: excluding the acute effect from baseline to week six and also by assessing slope from baseline to four-weeks off treatment. ${ }^{9,}{ }^{10} \mathrm{~A}$ third approach would be to leverage an active run-in period to assign different baseline GFRs to the active and control arms. In the MOSAIC trial, testing the effects of selonsertinib (which causes a negative acute 
effect by inhibiting creatinine secretion) in people with diabetic kidney disease, the GFR at the beginning of the run-in period is taken as the baseline measure for placebo-treated participants, while the GFR at randomization is used as the baseline measure in the active treatment arm. ${ }^{14}$ Finally, artificial censoring of GFR values after treatment discontinuation while using weighting or imputation to reduce the risk of selection bias may limit bias due to reversal of acute effects after treatment discontinuation. ${ }^{69}$ As we have previously shown, if negative acute effects are relatively small (e.g., no greater than $-1.5 \mathrm{~mL} / \mathrm{min} / 1.73 \mathrm{~m}^{2}$ ), adequate statistical power can be achieved to detect treatment effects using total slope if there is sufficient follow-up relative to the mean rate of progression in the study population. ${ }^{69}$

We observed positive acute effects primarily in studies of immunosuppressive agents. The cause is not well understood but it might be related to the early anti-inflammatory action of immunosuppressive agents in glomerular diseases, or the positive hemodynamic effect of steroids ${ }^{75}$. If an intervention has a positive acute effect but leads to harm on the longer term chronic slope, then positive acute effects could lead to false conclusions about treatment benefits if assessment of the treatment effect is performed on the total GFR slope. Bardoxolone produces an early increase in GFR which has been hypothesized to be due to an increase in mesangial surface area in addition to its longer term effect on reducing inflammation. ${ }^{76}$ Trials evaluating bardoxolone have computed change in GFR using off treatment GFR values to help determine whether the positive acute effect is a false conclusion or a lasting effect of the drug on the kidney. This underscores the importance of understanding the mechanisms for earlier vs longer term changes and designing trials accordingly.

The use of GFR slope to assess kidney disease progression may be particularly relevant to trials conducted in early stages of CKD. The association between larger negative acute effects and higher baseline mean GFR remained significant after adjustment for intervention and diabetes status. Thus, studies seeking to use GFR slope should carefully consider the mechanism of action of an 
intervention, study design and population characteristics (for example, the proportions of participants recruited with different levels of GFR), to ensure efficiency, and protect against the risk of a falsely positive or negative conclusion. Additionally, trials using GFR slope as an outcome must also ensure sufficient information on safety, given the time frames over which individuals with CKD are treated.

We observed a high degree of heterogeneity in the magnitude of acute effects, even within interventions where the nature and magnitude of the acute effect is anticipated due to well understood mechanisms of action. The reason for this observation is unclear. Possible variation in study quality may be one explanation of the heterogeneity; indeed there was greater heterogeneity among immunosuppressive studies which in general were smaller, and of lower quality. Because the acute effect was defined as the difference in change in GFR between treatment and control arms, differences in the care in the control arm (i.e., placebo vs active treatment) across different trials may have also contributed to the observed heterogeneity, although accounting for year of publication did not affect the results. Future work including newer studies with more consistent standard medical care in the control arm, may help to evaluate this hypothesis. In addition, as we previously demonstrated, combined assessment of changes in GFR and albuminuria may predict treatment effects and outcomes better than either alone. ${ }^{7}$ Further work will assess the utility of combined assessments of treatment effects on GFR slope and change in albuminuria that could overcome the challenges from acute effects.

This study has a number of strengths, including a systematic literature search, inclusion of a large number of studies with a diverse range of interventions, analyses of individual participant data, and the use of multiple methods to estimate acute GFR effects. However, a number of limitations should be considered when interpreting our findings. First, we estimated the acute effect at three months, but not all studies had measurements at this time point. For some studies the acute effect might 
have occurred over a different time period and might not be linear. For example, the negative acute effect with SGLT2 inhibition calculated at three months is less than previously reported at one month, possibly due to an attenuation of the acute effect over time. ${ }^{77,78}$ The timing of an acute effect is important to appreciate due to its implications for study power; as the timing of the acute effect increases, power to detect treatment effects on total slope decreases. Second, acute effects were calculated based on estimated GFR rather than measured GFR; while potentially important at an individual level, this is less likely to affect our conclusions at a population level. Third, while these analyses examined the impact of intervention type, GFR and UACR individually on the observed acute effects, impacts might be multifactorial and variation in acute effects could be due to other factors not captured. We also had relatively few studies for some interventions, including trials of SGLT2 inhibitors, and had no studies with paediatric participants. Finally, despite the large number of studies and participants overall, there were an insufficient number of trials to evaluate the magnitude of acute effects for individual classes of immunosuppressive agents with different mechanisms of action and to assess more granular subgroups, which could have explained the causes of the observed heterogeneity within these groups.

In summary, the magnitude and consistency of acute effects is variable across different interventions and may be larger at higher baseline GFR. Future work will involve understanding the timing of the acute effect as well as the associations of the acute effects with subsequent outcomes and longer-term treatments effects. Understanding the magnitude, timing and nature of the acute effect for a specific intervention and population can help inform the optimal design of randomized trials in CKD. 


\section{Author Contributions}

LA Inker, T Greene, HJL Heerspink, and EF Vonesh conceived of the study concept and design. TM Chan, FC Fervenza, J Floege, M Goicoechea, WG Herrington, E Imai, TH Jafar, JB Lewis, PKT Li, F Locatelli, BD Maes, RD Perrone, M Praga, A Perna, FP Schena, C Wanner, JFM Wetzels, M Woodward, and D Xie and their collaborators listed in Acknowledgments acquired the data. $\mathrm{H}$ Tighiouart, J Chaudhari, S Miao, E Vonesh, T Greene and LA Inker analyzed the data. All authors took part in the interpretation of the data. B Neuen, LA Inker and T Greene drafted the manuscript, and all authors provided critical revisions of the manuscript for important intellectual content. All collaborators who shared data were given the opportunity to comment on the manuscript. LA Inker, T Greene, and HJL Heerspink obtained funding for CKD-EPI while individual cohort support is listed in Appendix 2 in the Supplement. 


\section{Acknowledgments}

We thank all investigators, study teams, and patients for participating in the studies included in the meta-analysis. Study acronyms are listed in Appendix 1 in the Supplement along with other abbreviations. A variety of sources have supported the RCTs included in the CKD-EPI CT. These funding sources include government agencies such as national institutes of health and medical research councils as well as foundations and industry sponsors listed in Appendix 2 in the supplement.

CKD-EPI investigators/collaborators are listed here.

AASK: Tom Greene;

ABCD: Robert W. Schrier, Raymond O. Estacio;

ADVANCE: Mark Woodward, John Chalmers, Min Jun;

AIPRI (Maschio): Giuseppe Maschio, Francesco Locatelli;

ALTITUDE: Hans-Henrik Parving, Hiddo JL Heerspink;

Bari (Schena): Francesco Paolo Schena, Manno Carlo;

Bologna (Zucchelli): Pietro Zucchelli, Tazeen H Jafar;

Boston (Brenner): Barry M. Brenner;

canPREVENT: Brendan Barrett;

Copenhagen (Kamper): Anne-Lise Kamper, Svend Strandgaard;

CSG (Lewis 1992, 1993): Julia B. Lewis, Edmund Lewis;

EMPA-REG OUTCOME: Christoph Wanner, Maximilian von Eynatten;

Groningen (van Essen): Paul E. de Jong, GG van Essen, Dick de Zeeuw;

Guangzhou (Hou): Fan Fan Hou, Di Xie;

HALT-PKD: Arlene Chapman, Vicente Torres, Alan Yu, Godela Brosnahan;

HKVIN: Philip KT Li, Kai-Ming Chow, Cheuk-Chun Szeto, Chi-Bon Leung;

IDNT: Edmund Lewis, Lawrence G. Hunsicker, Julia B. Lewis; 
Lecco (Pozzi): Lucia Del Vecchio, Simeone Andrulli, Claudio Pozzi, Donatella Casartelli;

Leuven (Maes): Bart Maes;

Madrid (Goicoechea): Marian Goicoechea, Eduardo Verde, Ursula Verdalles, David Arroyo;

Madrid (Praga): Fernando Caravaca-Fontán, Hernando Trujillo, Teresa Cavero, Angel Sevillano;

MASTERPLAN: Jack FM Wetzels, Jan van den Brand, Peter J Blankestijn, Arjan van Zuilen;

MDRD Study: Gerald Beck, Tom Greene, John Kusek, Garabed Eknoyan;

Milan (Ponticelli): Claudio Ponticelli, Giuseppe Montagnino, Patrizia Passerini, Gabriella Moroni

ORIENT: Fumiaki Kobayashi, Hirofumi Makino, Sadayoshi Ito, Juliana CN Chan;

Hong Kong Lupus Nephritis (Chan): Tak Mao Chan;

REIN: Giuseppe Remuzzi, Piero Ruggenenti, Aneliya Parvanova, Norberto Perico;

RENAAL: Dick De Zeeuw, Hiddo JL Heerspink, Barry M. Brenner, William Keane;

ROAD: Fan Fan Hou, Di Xie;

Rochester (Donadio): James Donadio, Fernando C. Fervenza;

SHARP: Colin Baigent, Martin Landray, William Herrington, Natalie Staplin;

STOP-IgAN: Jürgen Floege, Thomas Rauen, Claudia Seikrit, Stefanie Wied;

Strasbourg (Hannedouche): Thierry P. Hannedouche;

SUN-MACRO: Julia B. Lewis, Jamie Dwyer, Edmund Lewis;

Texas (Toto): Robert D. Toto;

Victoria (Ihle): Gavin J. Becker, Benno U. Ihle, Priscilla S. Kincaid-Smith 


\section{Financial disclosures}

BL Neuen - travel support from Janssen and consultancy fees from Bayer and AstraZeneca, with all honoraria paid to his institution.

HJL Heerspink - grants and other from Abbvie, Janssen, Boehringer Ingelheim, AstraZeneca, other from Astellas, Fresenius, Gilead, and Merck outside the submitted work.

T Greene - grants from the NKF during the conduct of the study; and personal fees from DURECT Corporation, Janssen Pharmaceuticals, and Pfizer Inc., outside the submitted work.

EF Vonesh - served as a paid biostatistics consultant for the NKF for the expressed purpose of developing statistical models for use in the estimation and comparison of GFR slopes as a surrogate endpoint in CKD RCTs. In the past, he has also served as a biostatistics consultant to Prometic and Tricida, Inc., in which some of the work entailed consulting on the design and analysis of clinical trials in patients with CKD.

TM Chan - grants from Astellas, Baxter, and Kyowa Kirin.

F Fervenza - consultancy honoraria from Alexion, BioCryst, Morphosys, Novartis, Otsuka, GlaxoSmithKliine

J Floege - consultancy honoraria and/or speaker fees from Alnylam, Amgen, Astellas, AstraZeneca, Bayer, Calliditas, Fresenius, Omeros, Travere and Vifor

M Goicoechea - Consultancy honorary and/or speaker fees from Menarini, Genzyme, Alexion, NovoNordisk, Astra-Zeneca, Amgen, Vifor and Astellas.

W Herrington - supported by a Medical Research Council Kidney Research UK Professor David Kerr Clinician Scientist Award, and reports institutional grants from Boehringer Ingelheim, Eli Lilly, British Heart Foundation, HDR(UK). He adheres to a department policy not to accept honoraria or other payments from the pharmaceutical industry, expect for the reimbursement of costs to participate in scientific meetings.

E Imai - honoraria fees from Daiichi Sankyo, Boehringer Ingelheim and Bayer.

JB Lewis - principal investigator in study that contributed data to this analysis. Current consulting fees from CSL and BIOVIE

PKT Li - Speaker honorarium (personal fees) from Astra Zeneca, Baxter and FibroGen 
F Locatelli - personal fees from Amgen, Astellas, Bayer, Baxter, Norgine, Otzuka, Roche and Vifor Fresenius Pharma outside of the submitted work

B Maes - National Leader for the GSK Anaemia Studies in CKD: Erythropoiesis via a Novel PHI Daprodustat trial 807 and 808 and the National Leader for the Retrophin trial A Study of the Effect and Safety of Sparsentan in the Treatment of Patients With IgA Nephropathy.

RD Perrone - personal fees from Palladiobio, UpToDate KluwersWolter, Sanofi-Genzyme, and Otsuka $\mathrm{SA}$; and grants from Sanofi-Genzyme, Otsuka, Reata, and Kadmon outside of the submitted work.

M Praga - consultancy honoraria and/or speaker fees from Novartis, Alexion and Travere

FP Schena - grant from University of Bari

C Wanner - personal fees from Boehringer Ingelheim during the conduct of the study, and personal fees from Lilly, AstraZeneca, Bayer, and MSD outside the submitted work.

J Wetzels - grants from Sanofi, Pfizer, and Amgen; and other from Achillion, Shire, and Vifor Fresenius.

M Woodward - personal fees from Amgen, Kyowa Kirin and Freeline.

LA Inker - funding to institute for research and contracts with the National Institutes of Health, National Kidney Foundation, Retrophin, Omeros and Reata Pharmaceuticals; and consulting agreements with Tricida Inc. and Omeros Corp.

J Chaudhari, H Tighiouart, TH Jafar, A Perna and D Xie have no conflicts to disclose.

\section{Funding}

The study was funded by the National Kidney Foundation. 


\section{References}

1. Inker LA, Heerspink HJL, Tighiouart H, et al. GFR slope as a surrogate end point for kidney disease progression in clinical trials: A meta-analysis of treatment effects of randomized controlled trials. Journal of the American Society of Nephrology. 2019;30(9):1735-1745.

2. Grams ME, Sang Y, Ballew SH, et al. Evaluating glomerular filtration rate slope as a surrogate end point for ESKD in clinical trials: an individual participant meta-analysis of observational data. Journal of the American Society of Nephrology. 2019;30(9):1746-1755. 3. Heerspink HJL, Greene $\mathrm{T}$, Tighiouart $\mathrm{H}$, et al. Change in albuminuria as a surrogate endpoint for progression of kidney disease: a meta-analysis of treatment effects in randomised clinical trials. The lancet Diabetes \& endocrinology. 2019;7(2):128-139.

4. Coresh J, Heerspink HJL, Sang Y, et al. Change in albuminuria and subsequent risk of end-stage kidney disease: an individual participant-level consortium meta-analysis of observational studies. The lancet Diabetes \& endocrinology. 2019;7(2):115-127.

5. Coresh J, Turin TC, Matsushita K, et al. Decline in estimated glomerular filtration rate and subsequent risk of end-stage renal disease and mortality. Jama. 2014;311(24):25182531.

6. Levey AS, Inker LA, Matsushita K, et al. GFR decline as an end point for clinical trials in CKD: a scientific workshop sponsored by the National Kidney Foundation and the US Food and Drug Administration. American Journal of Kidney Diseases. 2014;64(6):821-835.

7. Levey AS, Gansevoort RT, Coresh J, et al. Change in albuminuria and GFR as end points for clinical trials in early stages of CKD: a scientific workshop sponsored by the National Kidney Foundation in collaboration with the US Food and Drug Administration and European Medicines Agency. American Journal of Kidney Diseases. 2020;75(1):84-104.

8. Thompson A, Smith K, Lawrence J. Change in Estimated GFR and Albuminuria as End Points in Clinical Trials: A Viewpoint From the FDA. American Journal of Kidney Diseases. 2020;75(1):4-5. doi:10.1053/j.ajkd.2019.08.007

9. Barratt J, Rovin B, Diva U, Mercer A, Komers R. Implementing the Kidney Health Initiative Surrogate Efficacy Endpoint in Patients With IgA Nephropathy (the PROTECT Trial). Kidney international reports. 2019;4(11):1633-1637. doi:10.1016/j.ekir.2019.08.007

10. Komers R, Diva U, Inrig JK, Loewen A, Trachtman H, Rote WE. Study Design of the Phase 3 Sparsentan Versus Irbesartan (DUPLEX) Study in Patients With Focal Segmental Glomerulosclerosis. Kidney international reports. 2020;5(4):494-502.

doi:10.1016/j.ekir.2019.12.017

11. US National Library of Medicine. ClinicalTrials.gov. Dabigatran vs Warfarin in AF Patients With T2D and CKD (RE-ELECT). Accessed May 27, 2020, https://clinicaltrials.gov/ct2/show/NCT03789695

12. US National Library of Medicine. ClinicalTrials.gov. Metformin vs Tolvaptan for Treatment of Autosomal Dominant Polycystic Kidney Disease (METROPOLIS). Accessed May 27, 2020, https://clinicaltrials.gov/ct2/show/NCT03764605

13. Meijer E, Drenth JP, d'Agnolo $\mathrm{H}$, et al. Rationale and design of the DIPAK 1 study: a randomized controlled clinical trial assessing the efficacy of lanreotide to Halt disease progression in autosomal dominant polycystic kidney disease. Am J Kidney Dis. Mar 2014;63(3):446-55. doi:10.1053/j.ajkd.2013.10.011 
14. US National Library of Medicine. ClinicalTrials.gov. Efficacy and Safety of Selonsertib in Participants With Moderate to Advanced Diabetic Kidney Disease (MOSAIC). Accessed May 27, 2020, https://clinicaltrials.gov/ct2/show/NCT04026165

15. Holtkamp FA, De Zeeuw D, Thomas MC, et al. An acute fall in estimated glomerular filtration rate during treatment with losartan predicts a slower decrease in long-term renal function. Kidney international. 2011;80(3):282-287.

16. Ohkuma $T$, Jun $M$, Rodgers $A$, et al. Acute increases in serum creatinine after starting angiotensin-converting enzyme inhibitor-based therapy and effects of its continuation on major clinical outcomes in Type 2 diabetes mellitus: The ADVANCE Trial. Hypertension. 2019;73(1):84-91.

17. Kraus BJ, Weir MR, Bakris GL, et al. Characterization and implications of the initial estimated glomerular filtration rate 'dip' upon sodium-glucose cotransporter-2 inhibition with empagliflozin in the EMPA-REG OUTCOME trial. Kidney International. 2021;99(3):750762.

18. Oshima M, Jardine MJ, Agarwal R, et al. Insights from CREDENCE trial indicate an acute drop in estimated glomerular filtration rate during treatment with canagliflozin with implications for clinical practice. Kidney international. Apr 2021;99(4):999-1009.

doi:10.1016/j.kint.2020.10.042

19. Kamper AL, Strandgaard S, Leyssac PP. Effect of enalapril on the progression of chronic renal failure. A randomized controlled trial. American journal of hypertension. Jul 1992;5(7):423-30.

20. Ihle BU, Whitworth JA, Shahinfar S, Cnaan A, Kincaid-Smith PS, Becker GJ.

Angiotensin-converting enzyme inhibition in nondiabetic progressive renal insufficiency: a controlled double-blind trial. Am J Kidney Dis. Apr 1996;27(4):489-95. doi:S0272-

6386(96)90158-4 [pii]

21. Hou FF, Zhang X, Zhang GH, et al. Efficacy and safety of benazepril for advanced chronic renal insufficiency. The New England journal of medicine. Jan 12 2006;354(2):13140. doi:354/2/131 [pii]

10.1056/NEJMoa053107

22. Hannedouche T, Landais P, Goldfarb B, et al. Randomised controlled trial of enalapril and beta blockers in non-diabetic chronic renal failure. BMJ. Oct 1 1994;309(6958):833-7.

23. Jafar TH, Schmid $\mathrm{CH}$, Landa $\mathrm{M}$, et al. Angiotensin-converting enzyme inhibitors and progression of nondiabetic renal disease. A meta-analysis of patient-level data. Annals of internal medicine. Jul 17 2001;135(2):73-87.

24. Maschio G, Alberti D, Janin G, et al. Effect of the angiotensin-converting-enzyme inhibitor benazepril on the progression of chronic renal insufficiency. The AngiotensinConverting-Enzyme Inhibition in Progressive Renal Insufficiency Study Group. The New England journal of medicine. Apr 11 1996;334(15):939-45.

25. Ruggenenti P, Perna A, Gherardi G, et al. Renoprotective properties of ACE-inhibition in non-diabetic nephropathies with non-nephrotic proteinuria. Lancet. Jul 31 1999;354(9176):359-64.

26. van Essen GG, Apperloo AJ, Rensma PL, et al. Are angiotensin converting enzyme inhibitors superior to beta blockers in retarding progressive renal function decline? Kidney Int Suppl. Dec 1997;63:S58-62.

27. Wright JT, Jr., Bakris G, Greene T, et al. Effect of blood pressure lowering and antihypertensive drug class on progression of hypertensive kidney disease: results from the AASK trial. JAMA. Nov 20 2002;288(19):2421-31. doi:joc20772 [pii] 
28. Brenner BM, Cooper ME, de Zeeuw D, et al. Effects of losartan on renal and cardiovascular outcomes in patients with type 2 diabetes and nephropathy. The New England journal of medicine. Sep 20 2001;345(12):861-9.

29. Imai E, Chan JC, Ito $S$, et al. Effects of olmesartan on renal and cardiovascular outcomes in type 2 diabetes with overt nephropathy: a multicentre, randomised, placebocontrolled study. Multicenter Study

Randomized Controlled Trial

Research Support, Non-U.S. Gov't. Diabetologia. Dec 2011;54(12):2978-86. doi:10.1007/s00125-011-2325-z

30. Lewis EJ, Hunsicker LG, Clarke WR, et al. Renoprotective effect of the angiotensinreceptor antagonist irbesartan in patients with nephropathy due to type 2 diabetes. The New England journal of medicine. Sep 20 2001;345(12):851-60. doi:10.1056/NEJMoa011303 31. Lewis EJ, Hunsicker LG, Lan SP, Rohde RD, Lachin JM. A controlled trial of plasmapheresis therapy in severe lupus nephritis. The Lupus Nephritis Collaborative Study Group. The New England journal of medicine. May 21 1992;326(21):1373-9.

32. Lewis EJ, Hunsicker LG, Bain RP, Rohde RD. The effect of angiotensin-convertingenzyme inhibition on diabetic nephropathy. The Collaborative Study Group. Clinical Trial Multicenter Study

Randomized Controlled Trial

Research Support, Non-U.S. Gov't

Research Support, U.S. Gov't, P.H.S. The New England journal of medicine. Nov 11 1993;329(20):1456-62. doi:10.1056/NEJM199311113292004

33. Packham DK, Wolfe R, Reutens AT, et al. Sulodexide fails to demonstrate renoprotection in overt type 2 diabetic nephropathy. Randomized Controlled Trial Research Support, Non-U.S. Gov't. J Am Soc Nephrol. Jan 2012;23(1):123-30. doi:10.1681/ASN.2011040378

34. Li PK, Leung CB, Chow KM, et al. Hong Kong study using valsartan in IgA nephropathy (HKVIN): a double-blind, randomized, placebo-controlled study. Am J Kidney Dis. May 2006;47(5):751-60. doi:10.1053/j.ajkd.2006.01.017

35. Torres VE, Abebe KZ, Chapman AB, et al. Angiotensin blockade in late autosomal dominant polycystic kidney disease. Multicenter Study

Randomized Controlled Trial

Research Support, N.I.H., Extramural

Research Support, Non-U.S. Gov't. New England Journal of Medicine. Dec 11 2014;371(24):2267-76. doi:10.1056/NEJMoa1402686

36. Schrier RW, Abebe KZ, Perrone RD, et al. Blood pressure in early autosomal dominant polycystic kidney disease. Multicenter Study

Randomized Controlled Trial

Research Support, N.I.H., Extramural

Research Support, Non-U.S. Gov't. New England Journal of Medicine. Dec 11 2014;371(24):2255-66. doi:10.1056/NEJMoa1402685

37. Parving HH, Brenner BM, McMurray JJ, et al. Cardiorenal end points in a trial of aliskiren for type 2 diabetes. Randomized Controlled Trial

Research Support, Non-U.S. Gov't. The New England journal of medicine. Dec 06 2012;367(23):2204-13. doi:10.1056/NEJMoa1208799 
38. Patel A, MacMahon S, Chalmers J, et al. Intensive blood glucose control and vascular outcomes in patients with type 2 diabetes. New England Journal Medicine. Jun 12 2008;358(24):2560-72. doi:NEJMoa0802987 [pii] 10.1056/NEJMoa0802987

39. Zucchelli P, Zuccala A, Borghi M, et al. Long-term comparison between captopril and nifedipine in the progression of renal insufficiency. Kidney international. Aug 1992;42(2):452-8.

40. Estacio RO, Jeffers BW, Gifford N, Schrier RW. Effect of blood pressure control on diabetic microvascular complications in patients with hypertension and type 2 diabetes. Diabetes care. Apr 2000;23 Suppl 2:B54-64.

41. Klahr S, Levey AS, Beck GJ, et al. The effects of dietary protein restriction and bloodpressure control on the progression of chronic renal disease. Modification of Diet in Renal Disease Study Group. The New England journal of medicine. Mar 31 1994;330(13):877-84.

42. Pozzi C, Andrulli S, Del Vecchio L, et al. Corticosteroid effectiveness in IgA nephropathy: long-term results of a randomized, controlled trial. J Am Soc Nephrol. Jan 2004;15(1):157-63.

43. Pozzi C, Andrulli S, Pani A, et al. Addition of azathioprine to corticosteroids does not benefit patients with IgA nephropathy. Multicenter Study

Randomized Controlled Trial. J Am Soc Nephrol. Oct 2010;21(10):1783-90.

doi:10.1681/ASN.2010010117

44. Pozzi C, Andrulli S, Pani A, et al. IgA nephropathy with severe chronic renal failure: a randomized controlled trial of corticosteroids and azathioprine. Randomized Controlled Trial. Journal of nephrology. Jan-Feb 2013;26(1):86-93. doi:10.5301/jn.5000110

45. Donadio JV, Jr., Grande JP, Bergstralh EJ, Dart RA, Larson TS, Spencer DC. The longterm outcome of patients with IgA nephropathy treated with fish oil in a controlled trial. Mayo Nephrology Collaborative Group. J Am Soc Nephrol. Aug 1999;10(8):1772-7.

46. Donadio JV, Jr., Larson TS, Bergstralh EJ, Grande JP. A randomized trial of high-dose compared with low-dose omega-3 fatty acids in severe IgA nephropathy. J Am Soc Nephrol. Apr 2001;12(4):791-9.

47. Rauen $T$, Eitner F, Fitzner $C$, et al. Intensive Supportive Care plus Immunosuppression in IgA Nephropathy. New England Journal of Medicine. 2015;373(23):2225-2236. doi:doi:10.1056/NEJMoa1415463

48. Maes BD, Oyen R, Claes K, et al. Mycophenolate mofetil in IgA nephropathy: results of a 3-year prospective placebo-controlled randomized study. Kidney international. May 2004;65(5):1842-9. doi:10.1111/j.1523-1755.2004.00588.x

49. Manno C, Torres DD, Rossini M, Pesce F, Schena FP. Randomized controlled clinical trial of corticosteroids plus ACE-inhibitors with long-term follow-up in proteinuric IgA nephropathy. Nephrology, dialysis, transplantation : official publication of the European Dialysis and Transplant Association - European Renal Association. Dec 2009;24(12):3694701. doi:10.1093/ndt/gfp356

50. Chan TM, Tse KC, Tang CS, Mok MY, Li FK. Long-term study of mycophenolate mofetil as continuous induction and maintenance treatment for diffuse proliferative lupus nephritis. J Am Soc Nephrol. Apr 2005;16(4):1076-84.

51. Ponticelli C, Zucchelli P, Passerini P, et al. A randomized trial of methylprednisolone and chlorambucil in idiopathic membranous nephropathy. The New England journal of medicine. Jan 5 1989;320(1):8-13. 
52. Ponticelli C, Zucchelli P, Passerini P, Cesana B. Methylprednisolone plus chlorambucil as compared with methylprednisolone alone for the treatment of idiopathic membranous nephropathy. The Italian Idiopathic Membranous Nephropathy Treatment Study Group. The New England journal of medicine. Aug 27 1992;327(9):599-603.

53. Ponticelli C, Altieri $\mathrm{P}$, Scolari F, et al. A randomized study comparing methylprednisolone plus chlorambucil versus methylprednisolone plus cyclophosphamide in idiopathic membranous nephropathy. J Am Soc Nephrol. Mar 1998;9(3):444-50.

54. Ponticelli $C$, Passerini $P$, Salvadori $M$, et al. A randomized pilot trial comparing methylprednisolone plus a cytotoxic agent versus synthetic adrenocorticotropic hormone in idiopathic membranous nephropathy. Am J Kidney Dis. Feb 2006;47(2):233-40.

55. Praga M, Barrio V, Juarez GF, Luno J. Tacrolimus monotherapy in membranous nephropathy: a randomized controlled trial. Kidney international. May 2007;71(9):924-30.

56. Zinman B, Wanner C, Lachin JM, et al. Empagliflozin, Cardiovascular Outcomes, and Mortality in Type 2 Diabetes. Comparative Study

Multicenter Study

Randomized Controlled Trial

Research Support, Non-U.S. Gov't. The New England journal of medicine. Nov 26 2015;373(22):2117-28. doi:10.1056/NEJMoa1504720

57. Goicoechea M, Garcia de Vinuesa S, Verdalles U, et al. Allopurinol and progression of CKD and cardiovascular events: long-term follow-up of a randomized clinical trial.

Randomized Controlled Trial. Am J Kidney Dis. Apr 2015;65(4):543-9.

doi:10.1053/j.ajkd.2014.11.016

58. Hou FF, Xie D, Zhang X, et al. Renoprotection of Optimal Antiproteinuric Doses (ROAD) Study: a randomized controlled study of benazepril and losartan in chronic renal insufficiency. J Am Soc Nephrol. Jun 2007;18(6):1889-98. doi:ASN.2006121372 [pii] 10.1681/ASN.2006121372

59. Peeters MJ, van Zuilen AD, van den Brand JA, et al. Nurse practitioner care improves renal outcome in patients with CKD. Randomized Controlled Trial

Research Support, Non-U.S. Gov't. J Am Soc Nephrol. Feb 2014;25(2):390-8.

doi:10.1681/ASN.2012121222

60. Barrett BJ, Garg AX, Goeree R, et al. A nurse-coordinated model of care versus usual care for stage $3 / 4$ chronic kidney disease in the community: a randomized controlled trial. Multicenter Study Randomized Controlled Trial

Research Support, Non-U.S. Gov't. Clinical journal of the American Society of Nephrology : CJASN. Jun 2011;6(6):1241-7. doi:10.2215/CJN.07160810

61. Baigent $C$, Landray MJ, Reith C, et al. The effects of lowering LDL cholesterol with simvastatin plus ezetimibe in patients with chronic kidney disease (Study of Heart and Renal Protection): a randomised placebo-controlled trial. Comparative Study

Randomized Controlled Trial

Research Support, Non-U.S. Gov't. Lancet. Jun 25 2011;377(9784):2181-92.

doi:10.1016/S0140-6736(11)60739-3

62. Higgins J, Green S. Cochrane Handbook for Systematic Reviews of Interventions. The Cochrane Collaboration; 2011. Updated Updated March 2011. Available from www.cochrane-handbook.org 
63. Praga M, Gutierrez E, Gonzalez E, Morales E, Hernandez E. Treatment of IgA nephropathy with ACE inhibitors: a randomized and controlled trial. J Am Soc Nephrol. Jun 2003;14(6):1578-83.

64. Frisch G, Lin J, Rosenstock J, et al. Mycophenolate mofetil (MMF) vs placebo in patients with moderately advanced IgA nephropathy: a double-blind randomized controlled trial. Nephrology, dialysis, transplantation : official publication of the European Dialysis and Transplant Association - European Renal Association. Oct 2005;20(10):2139-45.

65. Katafuchi R, Ikeda K, Mizumasa T, et al. Controlled, prospective trial of steroid treatment in IgA nephropathy: a limitation of low-dose prednisolone therapy. Am J Kidney Dis. May 2003;41(5):972-83. doi:S027263860300194X [pii]

66. Ruggenenti P, Perna A, Loriga $G$, et al. Blood-pressure control for renoprotection in patients with non-diabetic chronic renal disease (REIN-2): multicentre, randomised controlled trial. Lancet. Mar 12-18 2005;365(9463):939-46.

67. Levey AS, Stevens LA, Schmid $\mathrm{CH}$, et al. A new equation to estimate glomerular filtration rate. Annals of internal medicine. 2009;150(9):604-612.

68. Skali H, Uno H, Levey AS, Inker LA, Pfeffer MA, Solomon SD. Prognostic assessment of estimated glomerular filtration rate by the new Chronic Kidney Disease Epidemiology Collaboration equation in comparison with the Modification of Diet in Renal Disease Study equation. American heart journal. 2011;162(3):548-554.

69. Greene T, Ying J, Vonesh EF, et al. Performance of GFR Slope as a Surrogate End Point for Kidney Disease Progression in Clinical Trials: A Statistical Simulation. J Am Soc Nephrol. Sep 2019;30(9):1756-1769. doi:10.1681/asn.2019010009

70. Greene T, Teng CC, Inker LA, et al. Utility and validity of estimated GFR-based surrogate time-to-event end points in CKD: a simulation study. Research Support, N.I.H., Extramural

Research Support, Non-U.S. Gov't

Validation Studies. Am J Kidney Dis. Dec 2014;64(6):867-79. doi:10.1053/j.ajkd.2014.08.019

71. Inker LA, Heerspink HJL, Vonesh EF, Greene T. GFR Slope: Challenges and

Opportunities. Circulation Heart failure. 2021; In press

72. Oshima M, Neal B, Toyama T, et al. Different eGFR Decline Thresholds and Renal Effects of Canagliflozin: Data from the CANVAS Program. Journal of the American Society of Nephrology. 2020;31(10):2446-2456.

73. McCausland Finnian R, Lefkowitz Martin P, Claggett B, et al. Angiotensin-Neprilysin Inhibition and Renal Outcomes in Heart Failure With Preserved Ejection Fraction.

Circulation. 2020/09/29 2020;142(13):1236-1245.

doi:10.1161/CIRCULATIONAHA.120.047643

74. Jhund Pardeep S, Solomon Scott D, Docherty Kieran F, et al. Efficacy of Dapagliflozin on Renal Function and Outcomes in Patients With Heart Failure With Reduced Ejection Fraction. Circulation. 2021/01/26 2021;143(4):298-309.

doi:10.1161/CIRCULATIONAHA.120.050391

75. Baylis C, Handa RK, Sorkin M. Glucocorticoids and control of glomerular filtration rate. Seminars in nephrology. Jul 1990;10(4):320-9.

76. Baigent C, Lennon R. Should We Increase GFR with Bardoxolone in Alport Syndrome? Journal of the American Society of Nephrology. 2018;29(2):357.

doi:10.1681/ASN.2017101062

77. Wanner C, Heerspink HJL, Zinman B, et al. Empagliflozin and Kidney Function Decline in Patients with Type 2 Diabetes: A Slope Analysis from the EMPA-REG OUTCOME Trial. 
Journal of the American Society of Nephrology. 2018;29(11):2755.

doi:10.1681/ASN.2018010103

78. Neuen BL, Weldegiorgis M, Herrington WG, Ohkuma T, Smith M, Woodward M.

Changes in GFR and Albuminuria in Routine Clinical Practice and the Risk of Kidney Disease

Progression. Am J Kidney Dis. Sep 2021;78(3):350-360 e1. doi:10.1053/j.ajkd.2021.02.335 
Table 1. Clinical characteristics of the population overall and stratified by intervention

\begin{tabular}{|c|c|c|c|c|c|c|c|c|}
\hline & $\begin{array}{c}\mathbf{N} \\
\text { studies }\end{array}$ & $\underset{\text { participants }}{\mathbf{N}}$ & Age & Female & Black & Diabetes & GFR & ACR \\
\hline Overall & 53 & 56413 & $61.5(11.2)$ & $22514(37.4)$ & $4601(7.6)$ & $45342(75.3)$ & $61.8(26.3)$ & $59(13,539)$ \\
\hline \multicolumn{9}{|l|}{ Intervention } \\
\hline RASB vs & 19 & 25157 & $61.7(11.1)$ & 9964 (37.9) & $1720(6.5)$ & $22650(86.1)$ & $64.0(23.8)$ & $83(14,691)$ \\
\hline \multicolumn{9}{|l|}{ Control } \\
\hline RASB vs CB & 4 & 1884 & $57.6(9.1)$ & $832(36.3)$ & $862(37.6)$ & $1520(66.3)$ & $52.1(20.5)$ & $836(105,1983)$ \\
\hline $\mathrm{BP} *$ & 5 & 2438 & $51.0(12.8)$ & $1157(40.4)$ & 1235 (43.1) & 435 (15.2) & $55.9(26.3)$ & $68(24,393)$ \\
\hline Diet^$^{\wedge}$ & 2 & 731 & $51.8(12.4)$ & 332 (39.6) & 66 (7.9) & $43(5.1)$ & 34.5 (13.5) & $192(42,904)$ \\
\hline $\mathrm{IS}^{\#}$ & 15 & 1039 & 41.7 (12.9) & $407(33.0)$ & $19(1.5)$ & $4(0.3)$ & $72.1(29.2)$ & $1557(898,2814)$ \\
\hline Other & 8 & 25164 & $64.0(9.1)$ & $9822(36.8)$ & $699(2.6)$ & $20690(77.4)$ & $61.4(28.3)$ & $30(9,186)$ \\
\hline
\end{tabular}

Values for age and GFR are presented as mean (standard deviation) and for ACR as median $\left(25^{\text {th }}, 75^{\text {th }}\right.$

percentile). Values for other characteristics are presented as number (percentage). $\mathrm{N}$, sample size;

GFR, estimated glomerular filtration rate $\left(\mathrm{mL} / \mathrm{min} / 1.73 \mathrm{~m}^{2}\right) ; A C R$, albumin: creatinine ratio $(\mathrm{mg} / \mathrm{g})$;

$\mathrm{RASB}$, renin angiotensin system blockers; $\mathrm{CB}$, calcium channel blockers

*BP, low vs usual blood pressure control; ^Diet, low vs high protein diet; ${ }^{\#} I S$, various

immunosuppression treatment vs control studies

Note: The $\mathrm{N}$ participants presented here are for the primary analysis (ANCOVA). 
Figures and figure legends

Figure 1. Distribution and estimated mean acute effect on GFR by intervention

$\mathrm{Cl}$, confidence intervals; RASB, renin-angiotensin receptor blockers; $\mathrm{CCB}$, calcium channel blockers; $\mathrm{BP}$, blood pressure; SGLT2, sodium-glucose cotransporter-2; CKD, chronic kidney disease. Coverage interval refers to the interval under which $95 \%$ of the studies fall.

Figure 2: Meta regression of acute treatment effect on GFR by baseline GFR (top panel) and UACR (bottom panel)

Figure 3: Meta regression plot of variation in acute effect on GFR according to baseline GFR by intervention

Figure 4: Meta regression plot of variation in acute effect on GFR according to UACR by intervention

Figures 2 through 4: RASB, renin-angiotensin receptor blockers; CCB, calcium channel blockers; BP, blood pressure; SGLT2, sodium-glucose cotransporter-2; CKD, chronic kidney disease; UACR, urine albumin: creatinine ratio. 


\section{Supplemental Table of Contents}

Appendix 1: Abbreviations, units, and terms 2

Appendix 2: Study funding sources 3

Table S1. Search terms 5

Table S2. Study inclusion criteria

6

Table S3. Clinical characteristics of the population overall and stratified by disease

Table S4. Patient characteristics by study

8

Table S5. Estimated acute effects using different methods to compute the acute effect on GFR, overall and by intervention and disease 10

Table S6. Magnitude of acute effects on GFR, by intervention 11

Table S7. Multivariable meta-regression of acute effects on GFR, both for GFR and ACR 12

Figure S1. Evaluation of bias in studies included in meta-analysis 13

Figure S2. Mean baseline GFR and median baseline UACR across studies 15

Figure S3. Distribution and estimated mean acute effect on GFR by disease 16

Figure S4: Forest plot of acute effect on GFR by intervention, all studies, ANCOVA method

Figure S5: Forest plot of acute effects on GFR by intervention, all studies, sensitivity analysis using the linear mixed model 18

Figure S6: Variation in acute effect on GFR by year of study publication 19

Figure S7: Meta regression plot of variation in acute effect by (A) baseline natural log-transformed eGFR and (B) natural log acute effect by UACR 20

Figure S8: Meta regression plot of variation in acute effect by intervention by $(A)$ baseline natural log-transformed eGFR and (B) natural log acute effect by UACR 21

Figure S9: Variation in acute effect on GFR by within-study GFR quartiles, by intervention 